\title{
Effect of Fiber Reinforcement on the Response of Structural Members
}

\author{
G. Fischer
}

University of Hawaii, Honolulu, Hawaii, U.S.A..

V.C. Li

University of Michigan, Ann Arbor, Michigan, U.S.A.

\begin{abstract}
This paper describes a series of investigations concerning the effect of fiber reinforcement on the response of structural members in direct tension and flexure under reversed cyclic loading conditions. The synergistic effects of composite deformation mechanisms in the fiber reinforced cement composite (FRCC) and those in the structural member are identified. The FRCC used in the studies described herein is an Engineered Cementitious Composite (ECC) with strain hardening and multiple cracking properties in uniaxial tension and an ultimate tensile strain capacity on the order of several percent.
\end{abstract}

Keywords: fiber reinforcement, deformation compatibility, damage tolerance, structural response

\section{ENGINEERED CEMENTITIOUS COMPOSITES}

\subsection{Performance characteristics}

The deformation behavior of cementitious composites such as concrete, fiber reinforced concrete (FRC), and high performance fiber reinforced cement composites (HPFRCC) is typically distinguished according to their tensile stress-strain characteristics and post-cracking response in particular.

Brittle matrices, such as plain mortar and concrete, lose their tensile load-carrying capacity almost immediately after formation of the first matrix crack (Figure 1). The addition of fibers in conventional fiber reinforced concrete (FRC) can increase the toughness of cementitious matrices, however, their tensile strength and especially strain capacity beyond first cracking are not enhanced. FRC is therefore considered to be a quasi-brittle material with tension softening deformation behavior (Figure 1), i.e. a decaying load and immediate localization of composite deformation at first cracking in the FRC matrix.

ECC represents one particular class of HPFRCC, which are defined by an ultimate strength higher than their first cracking strength and the formation of multiple cracking during the inelastic deformation process (Figure 1) (Naaman and Reinhardt, 1995). In contrast to localized deformation in conventional FRC, where the apparent strain is dependent on the gage length, the deformation of ECC is uniform on a macro-scale and considered as pseudo-strain, which is a material property and independent of the gage length. ECC has typically an ultimate tensile strength of $5-8 \mathrm{MPa}$ and a strain capacity ranging from $3 \%$ to $5 \%$.

The spacing between multiple cracks in a typical ECC is on the order of several $\mathrm{mm}$, while the crack widths are limited to the order of $100 \mu \mathrm{m}$.

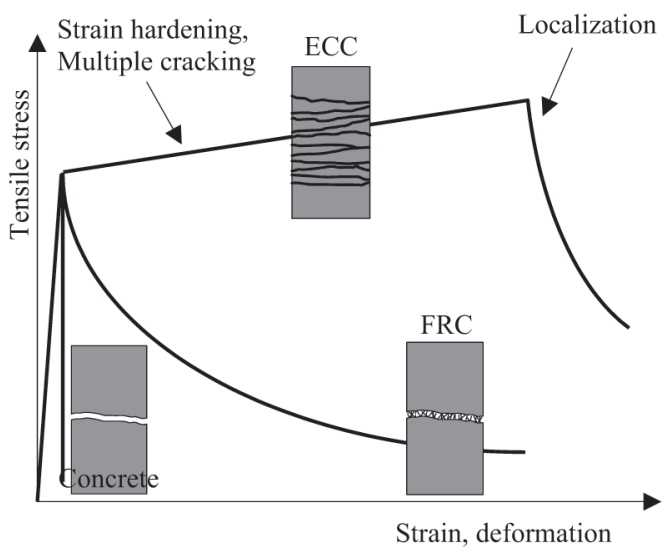

Figure 1 Tensile stress-strain behavior of cementitious matric 
Besides common ingredients of cementitious composites such as cement, sand, fly ash, water and additives, ECC utilizes short, randomly oriented polymeric fibers (e.g. Polyethylene, Polyvinyl Alcohol) at moderate fiber volume fractions $\left(\mathrm{V}_{\mathrm{f}}=1.5 \%-2 \%\right)$.

For structural applications in reinforced ECC members, processing of ECC requires conventional mixing equipment, such as a drum mixer, and can be adjusted to achieve regular consistency for casting and external compaction or a flowable consistency with self-compacting capabilities.

\subsection{Micromechanics-based design concept}

The main feature of ECC is the formation of multiple cracking at increasing composite tensile stress. This behavior hinges on two complementary requirements, specifically the peak bridging stress $\sigma_{\mathrm{B} \text {, peak }}$ exerted by the fibers at the cracked section must exceed the first cracking strength of the matrix $\sigma_{\mathrm{fc}}$, i.e.

$$
\sigma_{B, p e a k}>\sigma_{f c}
$$

such that the applied stress prior to matrix cracking can be carried by the fibers after matrix cracking. Furthermore, at formation of a matrix crack, propagation must occur at constant ambient stress $\sigma_{\mathrm{ss}}$ and constant crack opening $\delta_{\mathrm{ss}}($ Figure 2) in a flat crack configuration (Li and Leung, 1992). The latter condition results in an energy balance between the external work, the energy necessary to propagate the matrix crack, and the energy dissipated by the bridging fibers, i.e.

$$
\sigma_{s s} \delta_{s s}=G_{t i p}+\int_{0}^{\delta_{\text {ss }}} \sigma_{B}(\delta) d \delta
$$

where $\mathrm{G}_{\text {tip }}$ is the matrix toughness and $\delta$ is the crack opening.

The requirement that $\sigma_{\mathrm{ss}}<\sigma_{\mathrm{B} \text {,peak }}$ yields an upper limit for the matrix toughness

$$
G_{\text {tip }}<\sigma_{B, p e a k} \delta_{\text {peak }}-\int_{0}^{\delta_{s s}} \sigma_{B}(\delta) d \delta
$$

Equation (3) can be interpreted as follows: The complementary energy, defined as the energy supplied at maximum fiber bridging stress $\sigma_{\mathrm{B}}$, peak and corresponding crack opening $\delta_{\text {peak }}$ reduced by the energy consumed in fiber debonding and fiber pullout (right hand side of Equation (3)), must be sufficient to accommodate a steady state crack propagation, i.e. must exceed the matrix toughness at the crack tip $\mathrm{G}_{\text {tip }}$.

Some HPFRCC, which require fiber contents $\mathrm{V}_{\mathrm{f}}>5 \%$ and have considerable postcracking tensile strength, show limited tensile strain capacity prior to crack localization. In order to satisfy the above stated requirements (Equations (1) and (3)) at a minimum fiber volume fraction of a given fiber type, the properties of the cementitious matrix, the fiber, and the fiber/matrix interface must be considered. The micromechanical interaction of these constituents is the basis of design of engineered cementitious composites (ECC) as it affects the prerequisite mechanisms leading to steady state cracking and subsequent preservation of the composite load carrying capacity. Beyond formation of this particular type of crack, the characteristics of the stress-strain relationship of a given composite system are further governed by the bridging stress-crack opening relationship $\left(\sigma_{\mathrm{B}^{-}}\right.$ $\delta$ curve) and the flaw size distribution in the cementitious matrix.

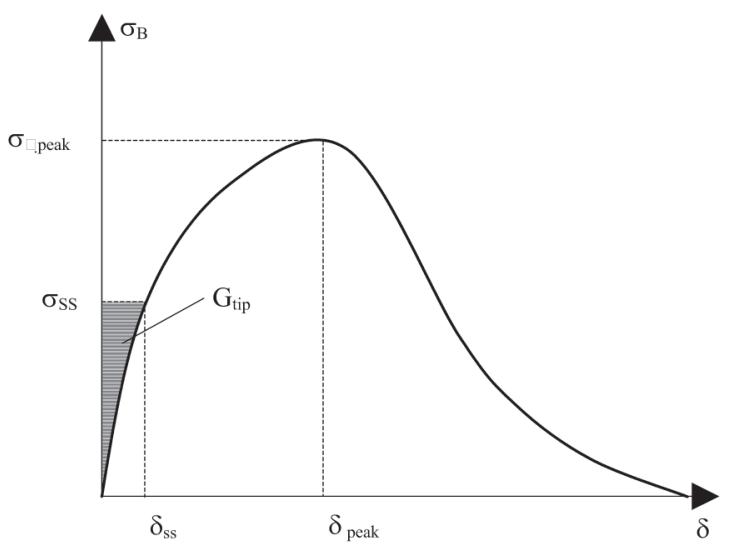

Figure $2 \sigma_{\mathrm{B}}-\delta$ curve and parameters for composite strain hardening

\subsection{Parameters affecting the $\sigma_{B}-\delta$ curve}

The $\sigma_{\mathrm{B}}-\delta$ curve results from the area-averaged load-crack opening $(\mathrm{P}-\delta)$ response of all fibers bridging a crack, which can be at different stages of interface debonding and fiber pullout depending on their position and orientation relative to the crack plane at a given crack opening.

The extraction of an individual fiber from the surrounding matrix occurs in a sequence of interfacial debonding and subsequent fiber pullout. The characteristics of this extraction process are strongly dependent on the type of fiber, in 
particular the nature of the interface between fiber and surrounding cementitious matrix. Interfacial debonding of a Polyethylene fiber (PE) is dominated by friction due to the hydrophobic nature of the fiber, while debonding of a Polyvinyl Alcohol (PVA) fiber is dominated by a strong chemical bond due to the hydrophilic nature of the fiber.

In case of Polyethylene (PE) fibers, the single fiber pullout curve (Figure 3 ) indicates exclusively frictional bond, i.e. immediate sliding of the debonded section of the fiber until the entire embedded fiber length is debonded. At constant interfacial friction, fiber pullout would occur at decreasing load corresponding to the decrease in contact area, however, scraping of the fiber surface increases the frictional resistance as the relative slip between fiber and surrounding matrix increases. Hence, beyond full debonding of the fiber, the applied load $\mathrm{P}$ continues to increase up to peak load and subsequently decreases until the fiber is completely pulled out. In order to fully utilize the tensile strength of the PE fiber and enhance the composite stress-strain behavior of PE-ECC, the interfacial bond strength is to be increased.

In case of PVA fibers, chemical bonding requires a certain load to initiate fiber extraction (Figure 3). Due to this dominant chemical bond, the fiber pullout load rapidly increases to a first peak in the $\mathrm{P}-\delta$ curve and is followed by a sudden load drop as debonding unstably propagates to the fiber end. Subsequently, friction dominates the pullout process, accompanied by a strong increase in frictional resistance due to fiber surface scraping. This results in a gradual reduction of

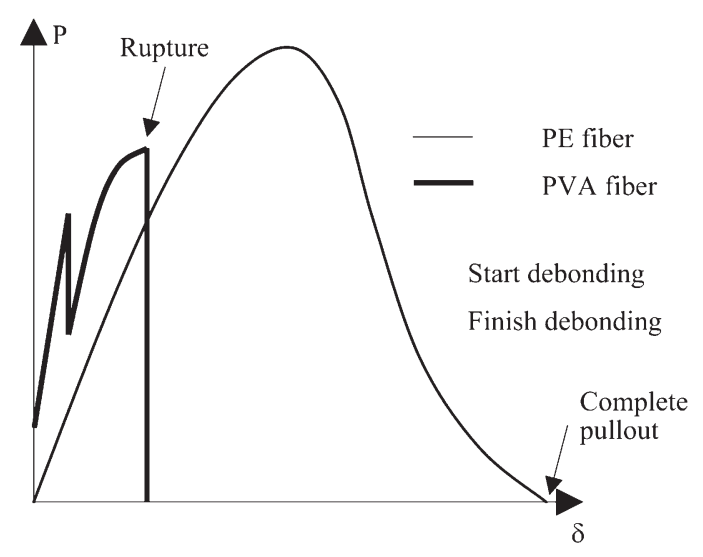

Figure 3 Schematic of single fiber pullout response fiber diameter and ultimate rupture of the fiber.

In essence, PE fiber has a relatively low frictional bond and reaches its maximum pullout load at relatively large pullout length, while PVA fiber has a high chemical bond and reaches its peak load at relatively small pullout length, however, does not completely pullout of the matrix but ruptures in the extraction process. In order to increase the opening of an individual crack and enhance the composite stress-strain behavior of PVA-ECC, the chemical and frictional bond of the PVA fiber is to be decreased, e.g. by means of particular surface treatment (Li et al., 2002) or by modification of the fiber/matrix interface transition zone. By controlling the fiber pullout processes, $\sigma_{\mathrm{B}}-\delta$ curves giving rise to high complementary energy have been achieved.

\section{EFFECT OF ECC ON DEFORMATION BEHAVIOR OF STRUCTURAL MEMBERS}

\subsection{Interaction of ECC and steel reinforcement}

Due to the particular material properties of ECC, a steel reinforced ECC member can be considered as a combination of a ductile cementitious matrix (ECC) and a reinforcing ductile element (steel). Evidence to support this approach can be obtained by investigating the deformation behavior of a steel reinforced ECC (R/ECC) member in uniaxial tension in contrast to that of conventional steel reinforced concrete $(\mathrm{R} / \mathrm{C})$.

The contribution of the cementitious matrix to the load-deformation response of reinforced concrete or ECC in uniaxial tension is generally described as tension-stiffening effect. The response of the reinforced cement composite is compared to that of the bare steel reinforcement and the difference is attributed to the tensile load carried by the cementitious matrix between transverse cracks.

Schematically, the difference in tensile loaddeformation response between $\mathrm{R} / \mathrm{C}$ and $\mathrm{R} / \mathrm{ECC}$ can be described using a representative composite element (Figure 4). Prior to reaching the first cracking strength of the cementitious matrix, the applied composite load is shared between reinforcement and matrix proportional to their stiffness and volume fraction. Stresses in both components are uniformly distributed in sections beyond the load transfer zone of the specimen. The formation of a transverse crack in the $\mathrm{R} / \mathrm{C}$ composite causes a redistribution of stresses in the 
matrix as well as in the reinforcement (Figure 4a). Since the concrete matrix is not able to transfer load across the crack, the applied load must be transferred to the reinforcement by bond action and is entirely carried by the reinforcement at the crack location. Due to the stress concentration in the reinforcement and the stress-free concrete matrix at the crack location, both materials experience a relatively large strain difference resulting in bond stresses and local slip. Consequently, composite deterioration can occur in various scenarios, such as interfacial bond failure, formation of inclined cracks originating from the interface, and longitudinal splitting due to radial pressure exerted by the ribs of the deformed reinforcing bar on the surrounding concrete.

The tensile ductility of the ECC matrix can on a macro scale eliminate the strain difference between reinforcement and matrix material. The $\mathrm{R} / \mathrm{ECC}$ member may be considered as a composite of two materials having elastic/plastic deformation behavior with individual yield strength and strain. As a result of these similar deformation characteristics, both constituents of the R/ECC composite are deforming compatibly in the elastic and inelastic deformation regime.

Cracking of ECC represents yielding of the matrix component while the steel reinforcement remains elastic. After cracking the stress distribution in the R/ECC composite is virtually unchanged (Figure 4b) since the stress in the ECC matrix at this instance remains constant and further increases with increasing deformation. In essence, the tensile load carried by the matrix prior to cracking is directly transferred (via bridging fibers) back to the uncracked parts of the matrix once the crack has formed. On a macro scale, bond stresses are not required to facilitate this transfer since load carried by the ECC matrix need not be transferred to the reinforcement. Due to the uniform stress in the cracked matrix, the distance between transverse cracks is a function of material properties of the fiber reinforced cement composite (ECC) and is independent of the interfacial bond properties between reinforcement and matrix. However, considering local effects in the immediate vicinity of one discrete crack in the ECC matrix, some interaction between reinforcement and matrix is expected. Depending on the micromechanical properties of the ECC matrix, a certain crack opening is required to develop a fiber bridging stress equal to that of the composite prior to cracking. Due to this microscopic discontinuity,

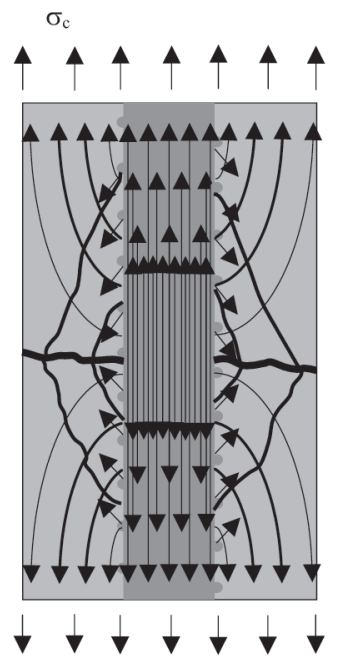
cracking a) $\mathrm{R} / \mathrm{C}$ after matrix

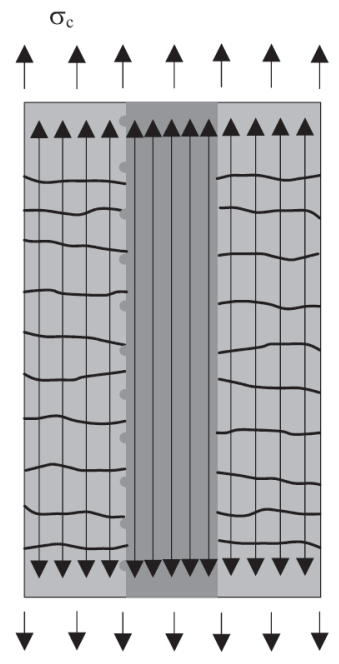

b) $\mathrm{R} / \mathrm{ECC}$ after matrix cracking
Figure 4 Crack formation and internal stresses in $\mathrm{R} / \mathrm{C}$ and $\mathrm{R} / \mathrm{ECC}$ composite

localized interfacial bond between steel reinforcement and ECC matrix is activated.

Yielding of the steel component constitutes the final deformation stage of the R/ECC member, where both constituent materials have entered the inelastic deformation regime. Strain-hardening deformation behavior of both components (steel and ECC) prevents localization of deformation at a particular section and compatible inelastic deformations of steel and ECC are maintained. Cracking of ECC as well as yielding of reinforcement is uniformly distributed over the length of the specimen. Because of the large volume of material involved in the inelastic deformation process, energy absorption is significantly enhanced. The fact that the ECC contribution to the load-carrying capacity can be maintained at relatively large deformation levels beyond steel yielding is directly attributed to the ductility of the ECC matrix, i.e. its multiple cracking deformation behavior.

These mechanisms in R/ECC composites have been experimentally verified and contrasted to the tension stiffening behavior of $\mathrm{R} / \mathrm{C}$ composites (Fischer and Li, 2002a). The comparison of the load-deformation response of $\mathrm{R} / \mathrm{ECC}$ and $\mathrm{R} / \mathrm{C}$ subjected to uniaxial tensile deformations clearly indicates the contribution of the ECC matrix to the load-carrying capacity particularly in the postcracking and post-yielding regime (Figure 5). 


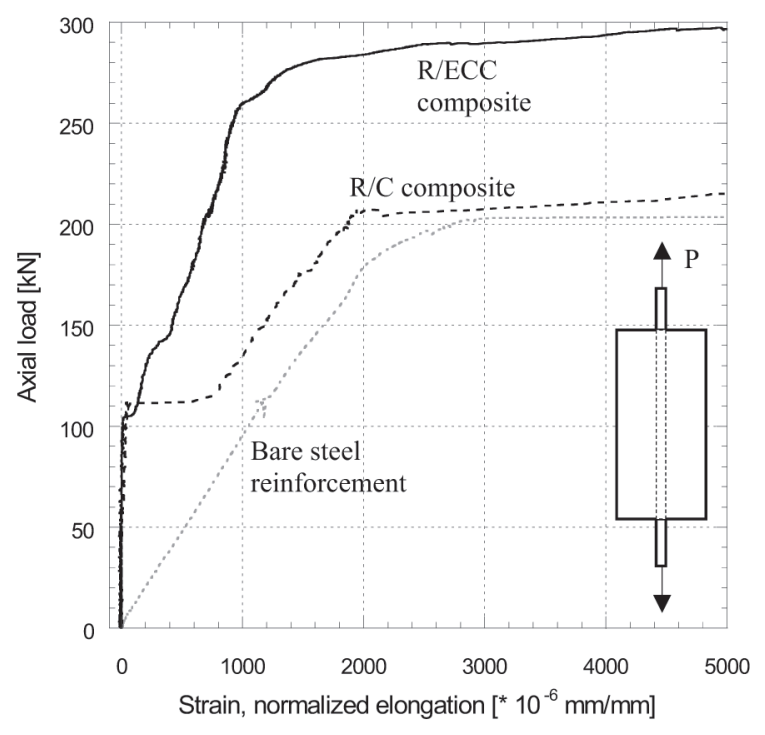

Figure 5 Axial load-deformation response of $\mathrm{R} / \mathrm{C}$ and $\mathrm{R} / \mathrm{ECC}$

Furthermore, the assumption of compatible deformations between ECC and steel reinforcement at large inelastic deformations is verified by observations on the interface between reinforcement and cementitious matrix after termination of the test. In the $\mathrm{R} / \mathrm{C}$ specimen, the interface between concrete and steel in the vicinity of the transverse crack is debonded and inclined cracking in the concrete matrix indicates the inability of concrete to accommodate the deformations induced by the steel reinforcement beyond yielding (Figure 6). In the R/ECC specimen, simultaneous yielding of steel and multiple cracking of ECC prevent the activation of
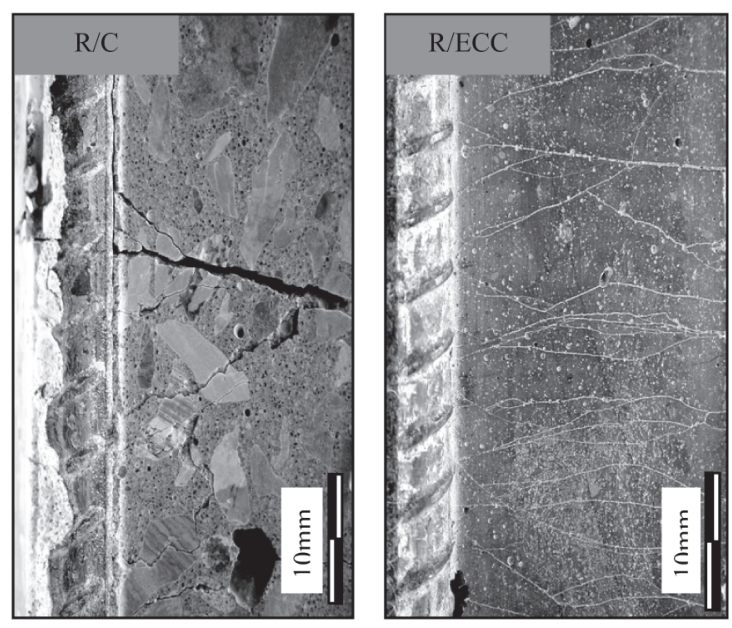

Figure 6 Interface condition beyond yielding of steel inforcement in $\mathrm{R} / \mathrm{C}$ and $\mathrm{R} / \mathrm{ECC}$ significant interfacial bond stress and consequently, the interface between steel reinforcement and ECC matrix remains intact throughout the elastic and inelastic deformation process of the R/ECC composite (Figure 6).

\subsection{Flexural deformation behavior of steel reinforced ECC members}

The preservation of composite integrity at relatively large deformations through the mechanisms described above for uniaxial tension has advantageous effects on the behavior of reinforced ECC flexural members especially under reversed cyclic loading conditions. Compatible deformation of ECC and longitudinal reinforcement will directly enhance the tensile component and indirectly ensure stable inelastic deformation on the compression side of the flexural member.

The performance of structures required to resist seismic excitations is dependent on the ability of selected structural components, in particular flexural members such as beams and columns in a moment resisting frame, to sustain relatively large inelastic deformations without significant loss of load carrying capacity. The ductility of these typical reinforced concrete components is indirectly dependent on the amount and configuration of transverse steel reinforcement, which serves as confinement of the concrete core and shear capacity enhancement and also provides resistance against buckling of longitudinal reinforcement.

Particularly under reversed cyclic loading conditions, the fundamental source of damage observed in reinforced concrete structures is the brittleness of concrete in general but in tension in particular. Structural deficiencies associated with this material property, such as bond splitting, concrete spalling, flexural strength decay due to shear failure, brittle compression failure and buckling of longitudinal reinforcement are usually overcome by arranging transverse reinforcement in order to confine concrete in compression or divert internal tensile forces from concrete to the transverse reinforcement to resist shear and prevent buckling of longitudinal reinforcement. Transverse reinforcement can be considered an external means to counteract internal material deficiencies of concrete to achieve a virtually ductile deformation behavior in tension and compression, with an increasing amount of transverse reinforcement 
resulting in increased structural ductility. Consequently, critical locations of structural elements, such as plastic hinge regions and joints, can be heavily congested and difficulties may arise in arranging the required amount of transverse reinforcement and in proper placement of concrete in these congested zones.

Despite enhanced resistance to undesirable failure modes by providing transverse reinforcement, the inherently brittle deformation behavior of concrete cannot be modified and deficiencies with respect to steel/concrete interaction, interfacial bond deterioration, and composite integrity are not overcome. While properly designed reinforced concrete structures ensure sufficient resistance to seismic excitations and satisfy primary safety requirements, research activities presented herein are motivated by the need to improve secondary performance requirements, such as reinforcement detailing requirements (potential reinforcement congestion and concrete compactability), construction feasibility and quality, damage tolerance, and repair needs, which are of significant economical concern.

The inelastic response of $\mathrm{R} / \mathrm{ECC}$ members under flexural load reversals is determined by the composite behavior in tension and compression, member shear resistance, matrix confinement effect, and resistance against buckling of longitudinal steel reinforcement. Considering the material properties of ECC and previous findings on the deformation mechanisms of $\mathrm{R} / \mathrm{ECC}$ in tension, the inelastic flexural response can be described by two conceptual stages before and after transition from multiple cracking to localization of cracking. The description of these stages will focus on the inelastic response of $\mathrm{R} / \mathrm{ECC}$, however, prior to yielding of steel reinforcement, the ductile deformation behavior of ECC will also affect the flexural member response by a more uniform distribution of flexural cracking with reduced crack spacing and individual crack widths compared to reinforced concrete composites.

Beyond yielding of steel reinforcement and prior to localization of cracking in the ECC matrix, a given displacement of the R/ECC flexural member is expected to require a reduced peak curvature in the plastic hinge region compared to the $\mathrm{R} / \mathrm{C}$ composite, resulting in reduced sectional demand on reinforcement tensile strain and compressive stress in ECC. This reduction of peak curvature is related to an extended distribution of deformation along the flexural member in particular beyond yielding of the longitudinal reinforcement (Figure 7). Similar to the composite deformation mechanism in uniaxial tension, the distribution of deformation is due to simultaneous strain-hardening of ECC and steel reinforcement. Besides reduced sectional demand, interfacial bond stresses are negligible due to compatible deformation between reinforcement and ECC and radial bond splitting forces are not generated. Consequently, longitudinal bond splitting cracks will not occur, which is expected to prevent interfacial bond deterioration, cover spalling and composite disintegration under tension and compression alternations. Thus, prior to localization of matrix cracking, the R/ECC member essentially benefits from a reduced sectional demand due to distributed flexural deformation along the specimen as opposed to localized crack formation observed in conventional $\mathrm{R} / \mathrm{C}$ members (Figure 7).

In the second stage, the strain capacity of ECC at the cantilever base is exhausted at a certain deflection level and localization of cracking leads to a concentration of deformation at this section. At this stage, the sectional demand is similar to reinforced concrete and consequently, deformation compatibility is lost and interfacial bond stresses are initiated. Slip between steel reinforcement and ECC causes radial stresses in the cementitious matrix, which in $\mathrm{R} / \mathrm{C}$ members lead to bond splitting and spalling of the concrete cover. In R/ECC, bond splitting cracks may occur beyond localization of flexural cracking in ECC, however, in the
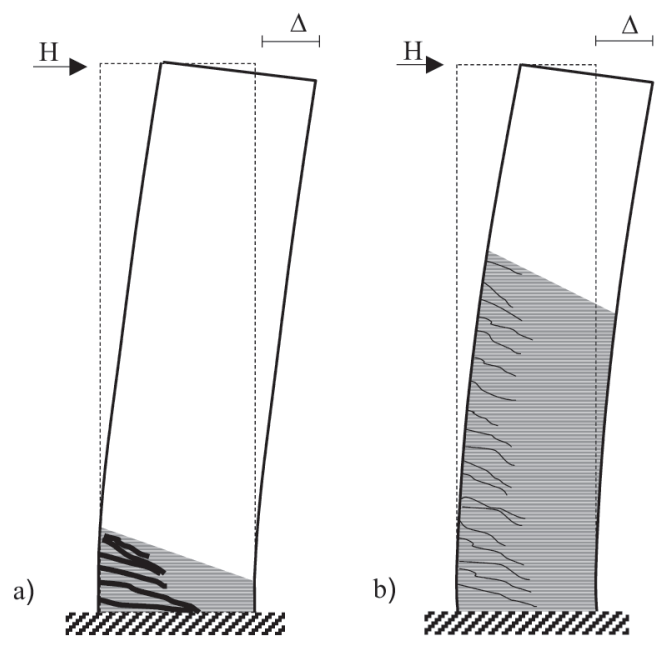

Figure 7 Idealized flexural deformation behavior of a) $\mathrm{R} / \mathrm{C}$ and b) R/ECC 
transverse direction ECC remains in the strainhardening regime with continuing resistance against cover spalling and reinforcement buckling. At this deformation stage, the R/ECC member benefits from the tensile strength of ECC beyond cracking, more specifically its confining effect and resistance against cover spalling.

Throughout both deformation stages, ECC is found to resist premature failure modes. Due to the intrinsic shear strength of ECC, additional transverse reinforcement provided by stirrups in potential plastic hinge regions and beyond may be significantly reduced. Moreover, the confinement effect of the ECC cover provides lateral resistance against buckling of steel reinforcement in the form of a continuous embedment similar to the effect of a confining jacket, which is additionally anchored into the ECC core by means of fiber bridging. The same mechanism also actively confines the ECC core, resulting in a ductile failure mode in compression.

With respect to structural ductility, the most important contribution of ECC to the structural response of the member is to maintain composite integrity and provide lateral stability for the reinforcing steel in order to endure cyclic inelastic deformations without buckling. Despite its considerable ductility in uniaxial tension, the cyclic behavior of ECC differs from that of a ductile metal, in that ECC is unable to recover its energy dissipation mechanism under alternating inelastic tensile and compressive deformations. Therefore, direct contributions of ECC to member flexural strength and energy dissipation are expected to be relatively small. However, its stabilizing effect on the longitudinal steel reinforcement and damage tolerance at large deformations are expected to considerably improve structural performance with respect to member energy dissipation capacity and damage evolution.

The load-deformation response of steel reinforced ECC members has been experimentally investigated and contrasted to a conventional $\mathrm{R} / \mathrm{C}$ member (Figure 8) (Fischer and Li, 2002b). The geometry, longitudinal reinforcement and loading configuration are identical in both specimens, while transverse steel reinforcement is provided in the $\mathrm{R} / \mathrm{C}$ specimen only. The comparison indicates performance improvements resulting from the ductile deformation behavior of ECC. In particular, the energy dissipation capacity of $\mathrm{R} / \mathrm{ECC}$ is significantly enhanced. The intrinsic shear capacity of ECC provides sufficient shear resistance for the reinforced member. Additional transverse steel reinforcement is ineffective and redundant in $\mathrm{R} / \mathrm{ECC}$ flexural members at given aspect ratio and low axial load levels.

Damage in R/ECC members is dominated by flexural cracking of ECC and stable inelastic deformations of steel reinforcement. ECC shows considerably higher damage tolerance than confined concrete. Bond splitting and spalling of ECC as well as composite disintegration due to cyclic loading are prevented (Figure 9).
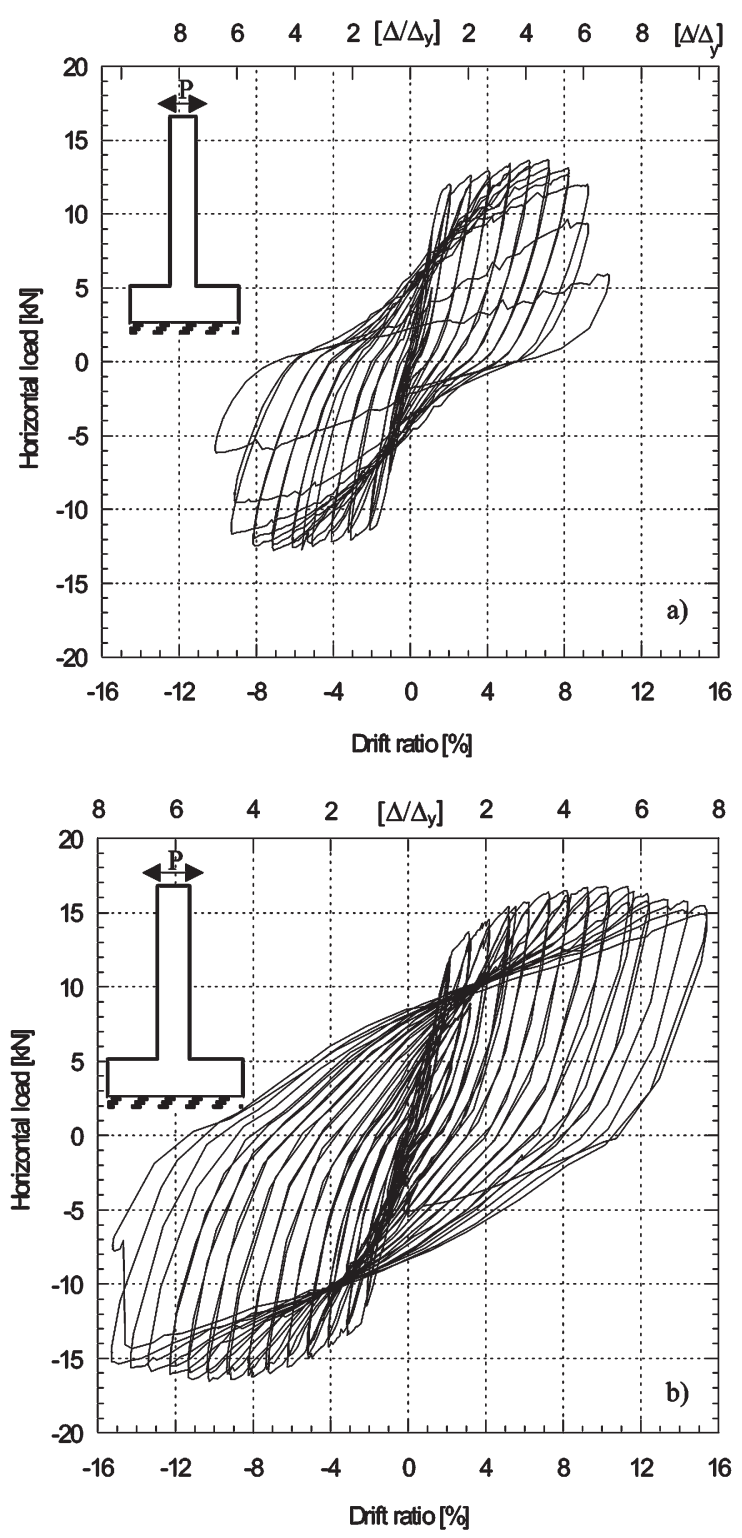

Figure 8 Load-deformation response of a) R/C and b) $\mathrm{R} / \mathrm{ECC}$ cantilever specimens with steel reinforcement 

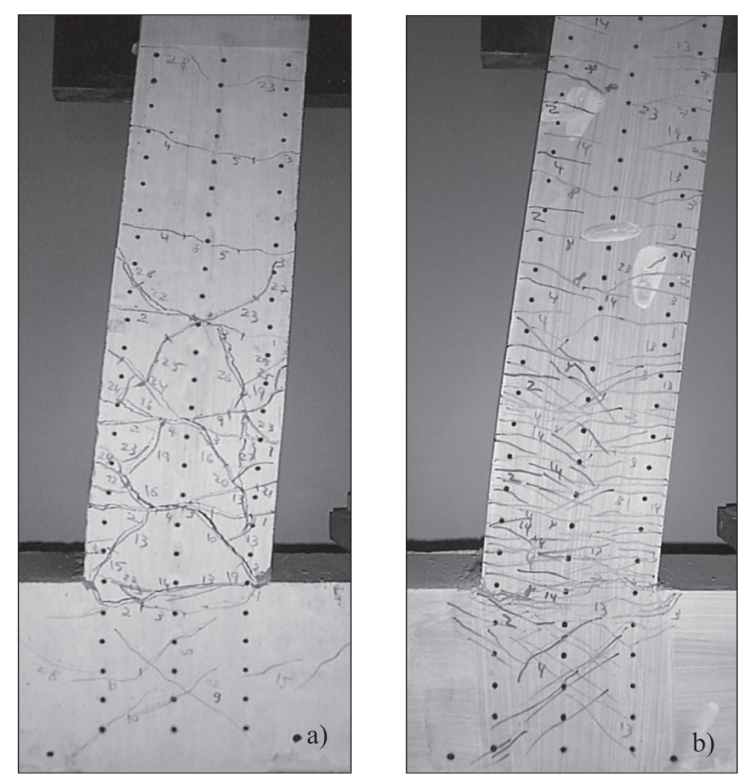

Figure 9 Deformed shape of a) R/C specimen and b) R/ECC specimen at respective peak load

\section{CONCLUSIONS}

The transition from ECC material properties to the behavior of structural members utilizing this material is fundamentally governed by the interaction of ECC and structural steel reinforcement in tension. Their similar elastic/plastic material properties lead to compatible deformations of both components in the elastic and inelastic deformation regime. Consequently, damage induced by local slip and excessive interfacial bond stress between steel reinforcement and cementitious matrix is prevented, resulting in improved performance of the reinforced ECC element in terms of axial loading capacity, ductility, and composite integrity. These synergistic effects are not primarily achieved by increased resistance of the materials in terms of tensile strength, confinement effect, or interfacial bond strength, but rather by reduced internal stresses and higher tolerance to damage typically observed in reinforced concrete structural members.

The material properties of ECC, in particular the extreme ductility, affect the tension stiffening effect of the cementitious composite. It is found that multiple cracking has a significant impact on the structural response resulting in compatible deformations between steel reinforcement and ECC in the inelastic deformation regime. While a considerable tension stiffening and strengthening effect is found in monotonic uniaxial tension, the contribution of ECC to the flexural strength of the reinforced structural member under cyclic loading conditions is found insignificant. Similarly, the extreme ductility of ECC is found to have no substantial direct contribution to the structural ductility, however, indirectly enhances the ductility of the member significantly.

For structural applications where concrete is substituted with ECC in particular or with FRCC in general, inelastic deformation capacity and damage tolerance rather than strength of the fiber reinforced cementitious composite are of paramount importance and should guide the composite design for structural performance. The addition of fiber reinforcement itself cannot be directly correlated to structural performance, but rather the material properties of ECC should be used to integrate materials design on the microscale with structural design on the macro-scale.

\section{REFERENCES}

Fischer, G. and Li, V.C., 2002a, "Influence of matrix ductility on the tension-stiffening behavior of steel reinforced Engineered Cementitious Composites (ECC)", ACI Structural Journal, Vol. 99, No.1, pp. 104-111, January-February

Fischer, G. and Li, V.C., 2002b, "Effect of matrix ductility on deformation behavior of steel reinforced ECC flexural members under reversed cyclic loading conditions", ACI Structural Journal, V.99, No.6, Nov.-Dec., pp. 781-790.

Li, V. C. and Leung, C.K.Y., 1992, "Steady-state and multiple cracking of short random fiber composites", Journal of Engineering Mechanics, ASCE, 118 (11), pp. 2246-2263

Li, V. C., C. Wu, S. Wang, A. Ogawa and T. Saito, "Interface Tailoring for Strain-hardening PVAECC," ACI Materials Journal, accepted, 2002.

Naaman, A.E. and Reinhardt, H.W., 1995, "Characterization of high performance fiber reinforced cement composites-HPFRCC", in Proc. of High Performance Fiber Reinforced Cement Composites 2 (HPFRCC 2), Ed. A.E. Naaman and H.W. Reinhardt, pp.1-23 